Acta Crystallographica Section B

Structural

Science

ISSN 0108-7681

David I. Woodward* and Ian M. Reaney

Ceramics and Composites Laboratory, Department of Engineering Materials, University of Sheffield, Sir Robert Hadfield Building, Mappin Street, Sheffield S1 3JD, England

Correspondence e-mail:

d.woodward@sheffield.ac.uk

\title{
Electron diffraction of tilted perovskites
}

Simulations of electron diffraction patterns for each of the known perovskite tilt systems have been performed. The conditions for the appearance of superlattice reflections arising from rotations of the octahedra are modified to take into account the effects of different tilt systems for kinematical diffraction. The use of selected-area electron diffraction as a tool for perovskite structure determination is reviewed and examples are included.

\section{Introduction}

Perovskites have been of major technological importance since Wul \& Goldman (1945) published research showing the extremely high relative permittivity of the perovskite $\mathrm{BaTiO}_{3}$. Most structural studies of perovskites are concerned with the mechanisms by which perovskites distort from their cubic prototype symmetry. One of these mechanisms is the rotating or 'tilting' of the corner-shared oxygen octahedra that form the structure. When an octahedron is tilted about one of the cubic $\langle 001\rangle$ directions, the four adjacent octahedra in the plane normal to the tilt axis are constrained to tilt in opposite senses. However, the octahedra directly above and below are not so constrained and can tilt in one of two ways. If they tilt in the same sense as the central octahedron, the tilt is described as being 'in-phase'. If they tilt in the opposite sense as the central octahedron, the tilt is described as being 'antiphase'.

Reaney et al. (1994) demonstrated the dependence of tilting on the tolerance factor, $t$, a quantity suggested by Goldschmidt (1926) to determine the stability of perovskite phases

$$
t=\left(R_{A}+R_{O}\right) /\left[2^{1 / 2}\left(R_{B}+R_{O}\right)\right],
$$

where $R_{A}, R_{B}$ and $R_{O}$ are the radii of the $A$ - and $B$-site ions and the $\mathrm{O}$ ion, respectively. The perovskite phase will form if $t$ is close to 1 . As $t$ decreases, the $A$-site ion becomes too small for the cuboctahedral site it occupies. When tilting occurs, it has the effect of reducing the volume of the interstice and improving the structural stability. By collating extensive structural data and calculating tolerance factors, Reaney et al. (1994) showed that at room temperature perovskites with $0.985<t<1.06$ are expected to have untilted structures. Perovskites with $0.964<t<0.985$ are usually tilted in antiphase and perovskites with $t<0.964$ are expected to show inphase and antiphase tilting. As $t$ continues to decrease, the stability of the perovskite phase decreases and eventually will not form.
Received 29 March 2005

Accepted 16 May 2005
C 2005 International Union of Crystallography Printed in Great Britain - all rights reserved 
A notation for describing and classifying tilting was proposed by Glazer (1972) and is widely used today. All tilts are described as combinations of component tilts about the three tetrad axes. The letters $a, b$ and $c$ are used to denote the magnitudes of tilt about the [100], [010] and [001] pseudocubic axes sequentially. Where the octahedra are tilted equally about two axes, this is denoted by repeating the appropriate letter; e.g. ' $a a a$ ' refers to equal tilts about all three axes and ' $a b c$ ' refers to unequal tilts about each axis. In-phase tilting is denoted in Glazer (1972) notation with a superscript '+' after the appropriate letter. Antiphase tilting is denoted with a superscript '-' after the appropriate letter. Where no tilting takes place about an axis, a superscript ' 0 ' is used. Hence, $a^{0} a^{0} c^{-}$refers to a perovskite with antiphase tilting about the pseudocubic $c$ axis only.

It was shown that 23 different tilt systems could be derived from this approach, but several of these result in the same space group and in fact only 15 distinct space groups were derived. Howard \& Stokes $(1998,2002)$ used a group-theoretical analysis to determine the possible tilt systems for perovskites, and also found 15 distinct space groups to describe the tilt systems. They further argued that there could only be 15 corresponding tilt systems, since if different tilt systems resulted in the same space group, then the system with the lowest symmetry must prevail. Howard \& Stokes (1998, 2002) represented the 15 space groups diagrammatically in order to indicate the group-subgroup relationship between tilt systems (Fig. 1). This diagram indicates the hierarchy of tilt systems by linking those systems that are related by an infinitesimal change in the tilt components.

Woodward (1997a) considered the geometrical implications of different tilt systems. He argued that for two of the space groups $\left(\mathrm{P}_{2} / \mathrm{nmc}\right.$ and $\left.\mathrm{Cmcm}\right)$, the octahedra may only remain connected if they distort. Howard \& Stokes $(1998,2002)$ later showed that distortion was not necessary for perovskites with the space group $\mathrm{Cmcm}$. Most treatments of tilting, including

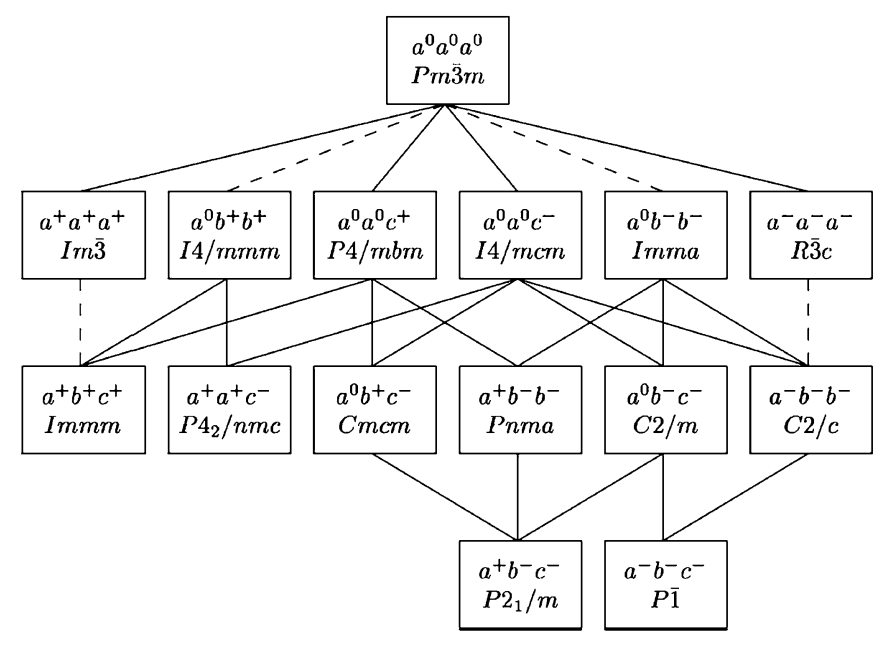

Figure 1

The distinct tilt systems and the group-subgroup relationship between them. Reproduced with kind permission from Howard \& Stokes (1998, 2002) that of Glazer (1972), assume perfectly rigid octahedra, but in practice some distortion of the octahedra always takes place. One interesting consequence of this is that the resultant space group may depend on how the octahedra distort, as demonstrated by Woodward (1997a) for the $a^{+} a^{+} a^{-}$and $a^{0} b^{+} b^{-}$tilt systems.

A large amount of current research is concerned with identifying the structures and symmetries adopted by tilted perovskites. X-ray diffraction is limited by the fact that scattering by oxygen is considerably weaker than that from cations and so the information concerning the oxygen sublattice is easily masked. Neutron diffraction from the oxygen sublattice is much stronger, relative to $\mathrm{X}$-rays, and this technique makes identifying distortions or rotations of the octahedra feasible. Nonetheless, small structural distortions which give rise to weak superlattice reflections may still be difficult to observe, particularly if they occur over relatively short coherence lengths. Electron diffraction has the advantage that singlecrystal/domain diffraction data may be routinely obtained from relatively small regions of $\sim 0.5 \mu \mathrm{m}$. It is also sensitive to superlattice reflections which arise due to weak, short-range effects. Therefore, knowledge of how different perovskite tilt systems relate to the electron diffraction patterns can make the unambiguous determination of symmetry easier.

\section{Methodology}

The work presented here is based on simulations of tilted perovskite structures using the appropriate space-group symmetry within the construction package of the computer program CaRIne Crystallography, Version 3.1. CaRIne is used to make basic structure-factor calculations and generate a reciprocal lattice with the appropriate kinematic intensities using the lattice parameters, space group symmetry and ionic positions. A slice of reciprocal space normal to low-order

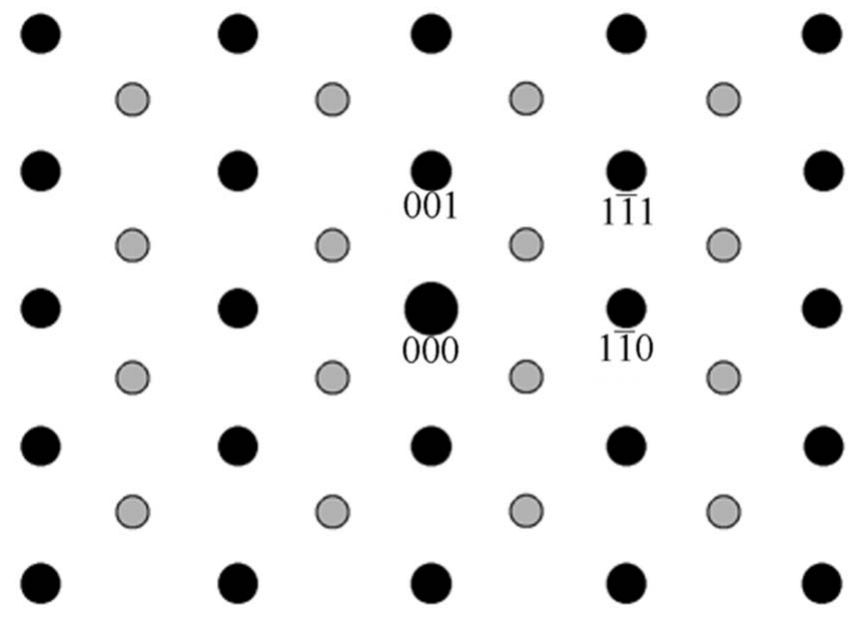

Figure 2

Simulation of experimental $\langle 110\rangle$ ZADP with fundamental reflections (black) and superstructure reflections arising from antiphase tilting (grey). Kinematically forbidden but doubly diffracted reflections are present. 
Table 1

List of conditions for allowed $\frac{1}{2}(o o o)$ reflections for all antiphase tilt systems.

\begin{tabular}{ll}
\hline Tilt system & Conditions for allowed reflections \\
\hline$a^{0} a^{0} a^{0}$ & No reflections \\
$a^{0} a^{0} c^{-}$ & $h \neq \pm k$ \\
$a^{0} b^{-} b^{-}$ & $k-l \neq 0\left(\right.$ or $k+l \neq 0$, depending on relative senses of $b^{-}$tilt $)$ \\
$a^{0} b^{-} c^{-}$ & No conditions \\
$a^{-} a^{-} a^{-}$ & $h \neq \pm k, k \neq \pm l, h \neq \pm l$ \\
$a^{-} b^{-} b^{-}$ & $k-l \neq 0\left(\right.$ or $k+l \neq 0$, depending on relative senses of $b^{-}$tilt $)$ \\
$a^{-} b^{-} c^{-}$ & No conditions \\
\hline
\end{tabular}

pseudocubic directions is then considered to represent major zone-axis diffraction patterns (ZADPs) from the perovskite crystal. No attempt was made to perform dynamical calculations. Consequently, intensities of reflections are only used qualitatively to aid interpretation of diffraction data. This approach is not unique and is a standard method of indexing electron diffraction data. However, care was taken to assess whether octahedra within a given structure were distorted or rotated without distortion.

The data set for the simulations was mainly generated from the ionic positions of structures previously determined by $\mathrm{X}$ ray and neutron diffraction. Where no known structure or compound corresponded to a specific tilt system, a model was created manually within the CaRIne software using the space groups suggested by Glazer (1972) and subsequently corrected by Glazer (1975) and Leinenweber \& Parise (1995). Small, sensible rotations of the octahedra were assumed, often using amplitudes of tilt and lattice parameters obtained from analogous compounds, and were used to generate non-zero superlattice intensities commensurate with the tilt system and space group. The computer program POTATO (Woodward, 1997b), which generates lattice parameters and ionic positions for tilted perovskites, was used to check these models.

The results of the simulations are intended to assist in using single-domain electron diffraction data to identify tilt systems and the associated symmetry. There is no attempt to model superlattice intensities for the different tilt systems using dynamical calculations. The authors feel that this is an unnecessary complication at this stage.

As the natures of in-phase and antiphase tilts are rather different, we will first consider solely antiphase tilt systems, followed by in-phase tilts and finally mixtures of the two. All crystallographic directions and planes refer to the simple pseudocubic unit cell.

\section{Antiphase tilt systems}

The problem with considering tilting as a combination of individual tilts about three Cartesian axes is, as stated by Glazer (1972), that the final structure depends on the order in which the tilts are imposed. The perovskite structure prediction programs POTATO (Woodward, 1997b) and SPUDS (Lufaso \& Woodward, 2001) deal with this problem by applying extremely small tilts incrementally to each of the three axes in turn. We, like Zhao et al. (1993), believe that the alternative is to consider antiphase tilting as a single operation about a chosen axis and that the tilt systems $a^{0} a^{0} c^{-}, a^{0} b^{-} b^{-}$ and $a^{-} a^{-} a^{-}$are special cases that involve tilting about the axes [001], [011] and [111], respectively, which pass through the centre of each octahedron.

Glazer (1975) gives simple formulae for determining the indices of the superstructure reflections that arise due to antiphase tilting, in addition to a formula for deriving the intensity of these reflections. These formulae indicate that antiphase tilting results in $\frac{1}{2}\{o o o\}$ reflections (where ' $o$ ' indicates an index with an odd number). Reaney et al. (1994) applied these rules to electron diffraction and showed that these reflections appear in $\langle 110\rangle$ ZADPs (Fig. 2).

The original diffraction rules proposed by Glazer (1975) for reflections produced as a result of antiphase tilting are reproduced here for convenience

$a^{-}$produces reflections of type $\frac{1}{2}(o o o)$ with $k \neq l$.

$b^{-}$produces reflections of type $\frac{1}{2}(o o o)$ with $h \neq l$.

$c^{-}$produces reflections of type $\frac{1}{2}(o o o)$ with $h \neq k$.

$$
I\left(a^{-}, b^{-}, c^{-}\right) \propto\left[\left(k i^{l}-l i^{k}\right) \alpha \pm\left(-l i^{h}+h i^{l}\right) \beta \pm\left(h i^{k}-k i^{h}\right) \gamma\right]^{2},
$$

where $h, k$ and $l$ are the Miller indices of the $\frac{1}{2}(h k l)$ reflection; $\alpha, \beta$ and $\gamma$ are the resolved tilt angles about [100], [010] and [001], respectively; $i=(-1)^{1 / 2}$.

Implicit within the intensity formula is the fact that certain reflections may become absent when tilting of equal magnitude occurs about different orthogonal axes. The conditions for allowed reflections have been detailed explicitly here in Table 1.

Electron diffraction does not, in general, result in perfectly kinematical scattering, since in only the thinnest sample will there be only one scattering event for an electron passing

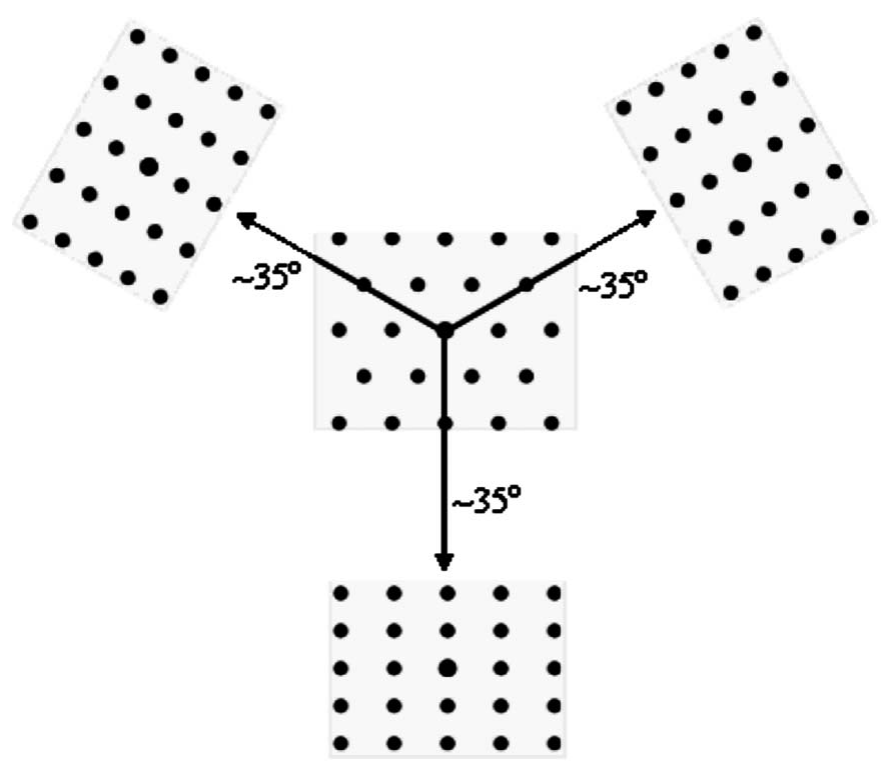

Figure 3

Orientation of $\langle 110\rangle$ zone axes relative to central $\langle 111\rangle$ axis. 
Table 2

The relationship between tilt axis and the occurrence of superstructure reflections in $\langle 110\rangle$ ZADPs for all different antiphase tilt systems.

\begin{tabular}{lll}
\hline Tilt system & Tilt axis & $\begin{array}{l}\text { Number of }\langle 110\rangle \text { zone axes containing } \\
\text { superstructure reflections (out of 12) }\end{array}$ \\
\hline$a^{0} a^{0} a^{0}$ & None & 0 \\
$a^{0} a^{0} c^{-}$ & $\langle 001\rangle$ & 8 \\
$a^{0} b^{-} b^{-}$ & $\langle 011\rangle$ & 10 \\
$a^{0} b^{-} c^{-}$ & $\langle 0 v w\rangle$ & 12 \\
$a^{-} a^{-} a^{-}$ & $\langle 111\rangle$ & 6 \\
$a^{-} b^{-} b^{-}$ & $\langle u v v\rangle$ & 10 \\
$a^{-} b^{-} c^{-}$ & $\langle u v w\rangle$ & 12 \\
\hline
\end{tabular}

through the sample. Multiple scattering is inevitable in most samples and this has the effect of giving reflections in positions in a diffraction pattern that are absent kinematically; e.g. in $a^{0} a^{0} c^{-}$tilting, the $\frac{1}{2}(111)$ reflection is kinematically forbidden from the [011] ZADP, but may occur by the double diffraction route: $\frac{1}{2}(311)+(100)$. Thus, the systematic absences of reflections cannot be applied to images of diffraction patterns as a means of identifying tilt systems.

The main reason for considering antiphase tilting as occurring about a single axis is critically because the resultant superstructure reflections are absent from the $\langle 110\rangle$ zone axes found perpendicular to the tilt axis. This may be derived from the intensity formula published by Glazer (1975), but has not been explicitly mentioned until recently (Woodward et al., 2003). In the case of the $a^{-} a^{-} a^{-}$tilt system, the tilt axis may be chosen as [111] and therefore 6 of the $12\langle 110\rangle$ variants contain
Table 3

The distribution of $\langle 110\rangle$ ZADPs containing superstructure reflections for antiphase tilt systems.

\begin{tabular}{llllll}
\hline & & \multicolumn{3}{l}{$\begin{array}{l}\text { No. of }\langle 110\rangle \text { ZADPs containing } \\
\text { superstructure reflections at } \sim 35^{\circ} \\
\text { from the }\langle 111\rangle \text { zone axes }\end{array}$} \\
\cline { 3 - 6 } Tilt system & Tilt axis (example) & {$[111]$} & {$[\overline{111}]$} & {$[1 \overline{1} 1]$} & {$[11 \overline{1}]$} \\
\hline$a^{0} a^{0} a^{0}$ & None & 0 & 0 & 0 & 0 \\
$a^{0} a^{0} c^{-}$ & {$[001]$} & 2 & 2 & 2 & 2 \\
$a^{0} b^{-} b^{-}$ & {$[011]$} & 3 & 3 & 2 & 2 \\
$a^{0} b^{-} c^{-}$ & {$[0 v w]$} & 3 & 3 & 3 & 3 \\
$a^{-} a^{-} a^{-}$ & {$[111]$} & 3 & 1 & 1 & 1 \\
$a^{-} b^{-} b^{-}$ & {$[u v v]$} & 3 & 3 & 2 & 2 \\
$a^{-} b^{-} c^{-}$ & {$[u v w]$} & 3 & 3 & 3 & 3 \\
\hline
\end{tabular}

superstructure reflections and the other six do not. In electron diffraction, this allows for either a statistical or an orientational method of deducing tilt systems. Table 2 lists the antiphase tilt systems, the corresponding tilt axes and the proportion of $\langle 110\rangle$ ZADPs that contain $\frac{1}{2}\{o o o\}$ reflections. These data can be used to progressively eliminate tilt systems simply by observing $\langle 110\rangle$ ZADPs, e.g. the presence of ZADPs both with and without superstructure reflections eliminates the tilt systems $a^{0} a^{0} a^{0}, a^{0} b^{-} c^{-}$and $a^{-} b^{-} c^{-}$.

The orientational method available for distinguishing between these systems has been previously demonstrated for $\mathrm{BiFeO}_{3}$ (Woodward et al., 2003). If a thin specimen is placed into a double tilt holder in a transmission electron microscope and a $\langle 111\rangle$ axis can be aligned parallel with the electron beam without significant tilting of the stage, then three zone axes of type $\langle 110\rangle$ may be obtained by tilting the stage through $\sim 35^{\circ}$ (Fig. 3). The presence or absence of superstructure reflections may then be used to infer the tilt system, using the information in Table 3. However, from Tables 2 and 3 it can be seen that this technique alone is insufficient to distinguish $a^{0} b^{-} b^{-}$ from $a^{-} b^{-} b^{-}$and neither can it distinguish $a^{0} b^{-} c^{-}$from $a^{-} b^{-} c^{-}$.

The latter two tilt systems are extremely uncommon in perovskites (Lufaso \& Woodward, 2001) and, in addition to $a^{-} b^{-} b^{-}$, may be considered as intermediate systems that are expected to occur only in narrow phase fields of solid solutions between more common, competing tilt systems, e.g. La-doped PZT (Knudsen et al., 2003). If tilting is viewed as an operation about a single axis, it is simple to envisage a continuous movement of the axis between two vectors. This is analogous to polarization, which, at the morphotropic phase boundary of $\mathrm{PbZr}_{x} \mathrm{Ti}_{1}{ }_{-x} \mathrm{O}_{3}$, is considered to rotate between [001]

Figure 4

Kinematical diffraction patterns simulated for perovskites with in-phase tilting about [001]. (a) The $\langle 100\rangle$ ZADP obtained parallel to the tilt axis, $(b)$ a $\langle 111\rangle$ ZADP with the unique $\langle 110\rangle$ axis indicated. Images $(c)$ and $(d)$ are simulations of the experimentally observed $\langle 100\rangle$ and $\langle 111\rangle$ zone-axis patterns, respectively, where multiple diffraction results in the appearance of additional reflections. 
(tetragonal) and [111] (rhombohedral) via an intermediate monoclinic phase in which the polarization vector lies within the common (110) plane (Noheda et al., 2000).

Movement of the tilt axis from [100] toward [111] within the (011) plane results in $a^{-} b^{-} b^{-}$tilting about an axis of type $[u v v]$, as would movement of the axis between [011] and [111]. Similarly, movement of an axis between [011] and [001] within the plane (100) would result in an $a^{0} b^{-} c^{-}$tilt system, whereas $a^{-} b^{-} c^{-}$tilting cannot result from any such simple movement of the axis between these principal directions.

The tilt systems $a^{-} b^{-} b^{-}, a^{0} b^{-} c^{-}$and $a^{-} b^{-} c^{-}$may be distinguished from all other antiphase tilt systems by the presence of superstructure reflections of varying intensity. In

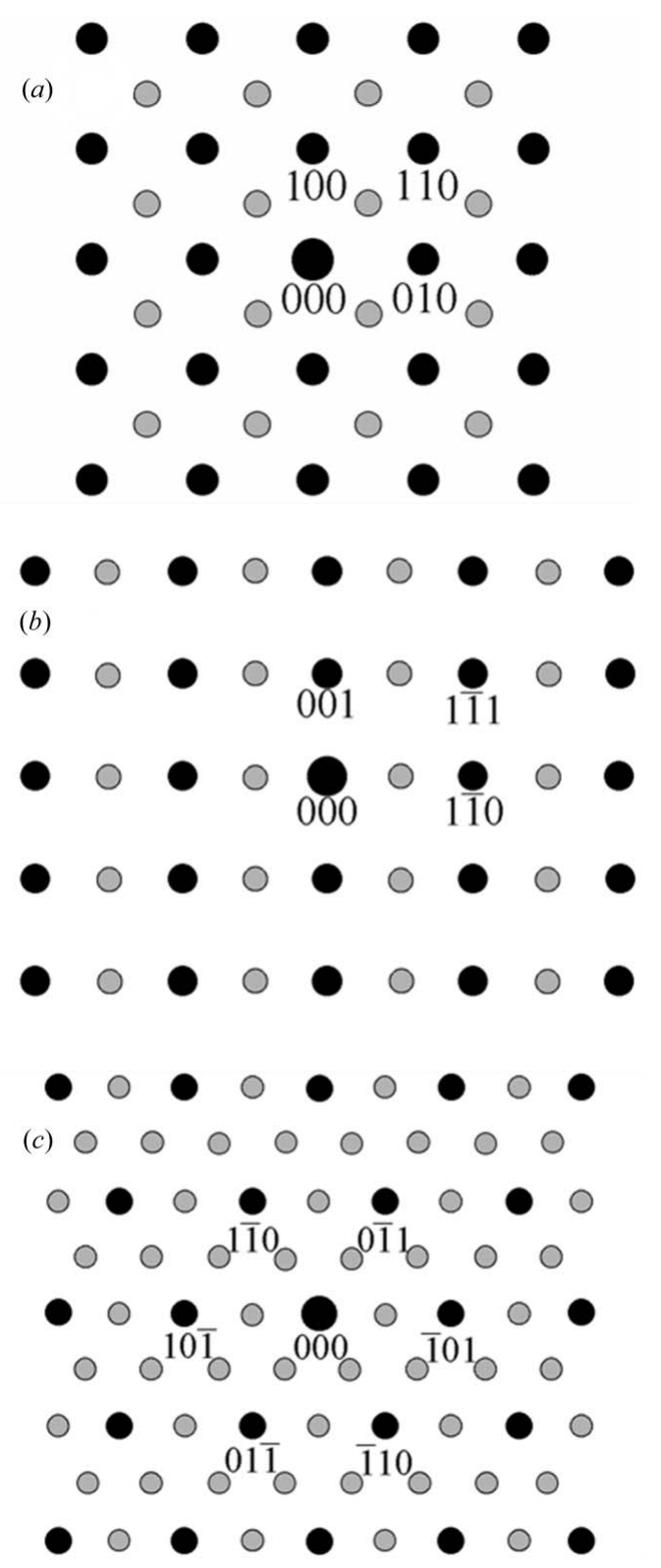

Figure 5

Diffraction patterns simulated for perovskites with in-phase tilting about more than one axis. (a) $\langle 100\rangle$ ZADP, (b) $\langle 110\rangle,(c)\langle 111\rangle$.
Table 4

The occurrence of superstructure reflections in $\langle 110\rangle$ ZADPs for all limiting cases of tilt system $a^{-} b^{-} b^{-}$.

\begin{tabular}{llll}
\hline & \multicolumn{3}{l}{$\begin{array}{l}\text { Number of }\langle 110\rangle \text { zone axes containing superstructure } \\
\text { reflections of specific intensity (out of 12) }\end{array}$} \\
\cline { 2 - 4 } Limiting case & Strong & Weak & Absent \\
\hline$a \simeq b$ & 6 & 4 & 2 \\
$a \simeq 0$ & $10 \dagger$ & 0 & 2 \\
$b \simeq 0$ & 8 & 2 & 2 \\
\hline
\end{tabular}

$\dagger$ Intensities are all strong, but not constrained to be equal.

Table 5

The distribution of $\langle 110\rangle$ ZADPs containing superstructure reflections for the three limiting cases of the $a^{-} b^{-} b^{-}$tilt system.

\begin{tabular}{|c|c|c|c|c|c|}
\hline \multirow[b]{2}{*}{ Limiting case } & \multirow[b]{2}{*}{ Tilt pseudo-axis } & \multicolumn{4}{|c|}{$\begin{array}{l}\text { No. of }\langle 110\rangle \text { ZADPs containing } \\
\text { superstructure reflections at } \sim 35^{\circ} \text { from } \\
\langle 111\rangle \text { zone axes in the order } \\
\text { strong/weak/absent }\end{array}$} \\
\hline & & [111] & [1111] & [11̄1] & {$[11 \overline{1}]$} \\
\hline$a \simeq b$ & {$[111]$} & $3 / 0 / 0$ & $1 / 2 / 0$ & $1 / 1 / 1$ & $1 / 1 / 1$ \\
\hline$a \simeq 0$ & [011] & $3 / 0 / 0$ & $3 / 0 / 0$ & $2 / 0 / 1$ & $2 / 0 / 1$ \\
\hline$b \simeq 0$ & {$[100]$} & $2 / 1 / 0$ & $2 / 1 / 0$ & $2 / 0 / 1$ & $2 / 0 / 1$ \\
\hline
\end{tabular}

all other antiphase tilt systems, the superstructure reflections are constrained to be of equal intensity due to the zone axes all being inclined at equal angles to the tilt axis. In the case of $a^{-} b^{-} b^{-}$and $a^{0} b^{-} c^{-}$systems, the reflections may be qualitatively categorized as either weak or strong, relative to the fundamental reflections. The ability to separate reflections into weak and strong provides additional structural information, especially if the statistical or geometric distribution of the different zone axis types can be determined. A note of caution should be sounded here, since the intensity of reflections may be affected by physical factors, in particular the thickness of the sample, and great care should be taken not to place too much emphasis on slight differences in intensity. The cases we will consider here will be those where the superstructure reflections are either strong, extremely weak or completely absent.

\subsection{The $a^{-} b^{-} b^{-}$tilt system}

This tilt system may be found as an intermediate when the tilt axis moves in one of two ways within the (011) plane:

(i) $[011] \leftrightarrow[111]\left(35.3^{\circ}\right)$;

(ii) $[100] \leftrightarrow[111]\left(54.7^{\circ}\right)$.

(A tilt axis moving between [100] and [011] must pass through [111] and is therefore incorporated into the two cases above).

Three extreme cases of this tilt system can be derived: $a \simeq b$, where the tilt axis approaches [111]; $a \simeq 0$, where the axis approaches [011]; $b \simeq 0$, where the axis approaches [100]. Tables 4 and 5 show how the distributions of superstructure reflections change between the limiting cases.

The data in Tables 4 and 5 show that $a^{-} b^{-} b^{-}$tilt systems in the limiting cases $a \simeq b$ and $b \simeq 0$ can be distinguished from 
Table 6

The occurrence of superstructure reflections in $\langle 110\rangle$ ZADPs for both limiting cases of tilt system $a^{0} b^{-} c^{-}$.

\begin{tabular}{llll}
\hline & \multicolumn{2}{l}{$\begin{array}{l}\text { Number of }\langle 110\rangle \text { zone axes containing superstructure } \\
\text { reflections of specific intensity (out of 12) }\end{array}$} \\
\cline { 2 - 4 } Limiting case & Strong & Weak & Absent \\
\hline$b \simeq c$ & 10 & 2 & 0 \\
$b \simeq 0$ & 8 & 4 & 0 \\
\hline
\end{tabular}

each other, but distinguishing the limiting case $a \simeq 0$ from the tilt system $a^{0} b^{-} b^{-}$by electron diffraction is made impossible due to the absence of weak superstructure reflections.
Table 7

The distribution of $\langle 110\rangle$ ZADPs containing superstructure reflections for both limiting cases of the $a^{0} b^{-} c^{-}$tilt system.

\begin{tabular}{|c|c|c|c|c|c|}
\hline \multirow[b]{2}{*}{ Limiting case } & \multirow[b]{2}{*}{ Tilt pseudo-axis } & \multicolumn{4}{|c|}{$\begin{array}{l}\text { No. of }\langle 110\rangle \text { ZADPs containing } \\
\text { superstructure reflections at } \sim 35^{\circ} \text { from } \\
\langle 111\rangle \text { zone axes in the order } \\
\text { strong/weak/absent }\end{array}$} \\
\hline & & [111] & {$[\overline{1} 11]$} & {$[1 \overline{1} 1]$} & {$[11 \overline{1}]$} \\
\hline$b \simeq c$ & [011] & $3 / 0 / 0$ & $3 / 0 / 0$ & $2 / 1 / 0$ & $2 / 1 / 0$ \\
\hline$b \simeq 0$ & [001] & $2 / 1 / 0$ & $2 / 1 / 0$ & $2 / 1 / 0$ & $2 / 1 / 0$ \\
\hline
\end{tabular}

\subsection{The $a^{0} b^{-} c^{-}$tilt system}

This tilt system is found as an intermediate when the tilt axis moves between [011] and [010] or [001] in the (100) plane through an angle of $45^{\circ}$. In this case there are two distinct limiting cases; $b \simeq c$ and $b($ or $c) \simeq 0$.

The data in Tables 6 and 7 may assist in identifying the $a^{0} b^{-} c^{-}$tilt system. However, in all but the limiting cases described above, this may easily be confused with the $a^{-} b^{-} c^{-}$system, which similarly produces superstructure reflections in all $\langle 110\rangle$ ZADPs, but without any constraints on the intensity of these reflections. An extensive search by Woodward (1997c) reveals only one perovskite with an $a^{-} b^{-} c^{-}$tilt, so it is not common. Selected-area electron diffraction of a single sample is therefore not an adequate way to determine unambiguously the presence of this tilt system.

\section{In-phase tilt systems}

In-phase tilt systems differ subtly from antiphase tilt systems. The principle of considering a single tilt axis, as performed for antiphase tilting, does not apply to in-phase tilt systems, but as there are only four, this is not much of a problem. The $a^{0} a^{0} c^{+}$system is simple and easily derived. A treatment of $a^{+} a^{+} a^{+}$and $a^{0} b^{+} b^{+}$systems has been performed by O'Keeffe \& Hyde (1977).

Glazer (1975) developed rules for superstructure reflections arising from in-phase tilting. Again, these are reproduced here for convenience
Figure 6

Electron diffraction patterns obtained from a sample of $\mathrm{CaTiO}_{3}$. These are arbitrarily indexed with zone axes $(a)$ [100], (b) [010], (c) [110], (d) [101] and (e) [111]. Superstructure reflections are indicated with: 'a' $=$ antiphase tilt reflection, ' $\mathrm{i}$ ' $=$ in-phase tilt reflection, ' $c$ ' $=$ concert reflection/antiparallel cation displacement. 
$a^{+}$produces reflections of type $\frac{1}{2}(e o o)$ with $k \neq l$

$b^{+}$produces reflections of type $\frac{1}{2}($ oeo $)$ with $h \neq l$

$c^{+}$produces reflections of type $\frac{1}{2}($ ooe $)$ with $h \neq k$.

Adaptation by Reaney et al. (1994) of these rules for electron diffraction showed that these reflections appear in $\langle 100\rangle$ and $\langle 111\rangle$ zone axes and are absent from the $\langle 110\rangle$ zone axes for the tilt systems studied.

Simulations of in-phase tilted perovskites have shown that the above conditions are only true in the case where a single axis is in-phase tilted. The $a^{0} a^{0} c^{+}$tilt system exhibits superstructure reflections in only the $\langle 001\rangle$ ZADP obtained parallel to the tilt axis and also in all $\langle 111\rangle$ ZADPs, consistent with the conditions listed by Glazer (1975). Fig. 4 shows schematics of the appropriate diffraction images. The arrangement of superstructure reflections in the $\langle 111\rangle$ ZADPs destroys the sixfold symmetry of the diffraction pattern, giving rise to only twofold symmetry and a unique $\langle 110\rangle$ direction. This direction is perpendicular to the in-phase tilt axis; e.g. in Fig. $4(b)$ the unique axis is [110] or [110], perpendicular to the in-phase tilt axis [001].

However, when more than one axis is tilted in-phase, these rules change. Reflections of the type $\frac{1}{2}\{o o e\}$ are still generated, but without any of the constraints listed above. As a result, $\frac{1}{2}\{$ ooe $\}\left[\right.$ i.e. $\frac{1}{2}($ ooe $), \frac{1}{2}($ oeo $)$ and $\frac{1}{2}($ eoo $\left.)\right]$ superstructure reflections are found to be present in all $\langle 100\rangle,\langle 110\rangle$ and $\langle 111\rangle$ ZADPs in all positions. Fig. 5 shows the schematics of these zone axes.

Selected-area electron diffraction cannot distinguish between two axes tilted in-phase and three axes tilted inphase. The tilt systems $a^{+} a^{+} a^{+}, a^{0} b^{+} b^{+}$and $a^{+} b^{+} c^{+}$all produce the same number, type and distribution of superstructure reflections. This is immediately obvious to the reader when it is considered that the space groups for these tilt systems are $\operatorname{Im} \overline{3}$, $I 4 / \mathrm{mmm}$ and Immm, respectively. The symmetry operators in each space group produce the same reflection conditions, dominated by the body-centred operation.

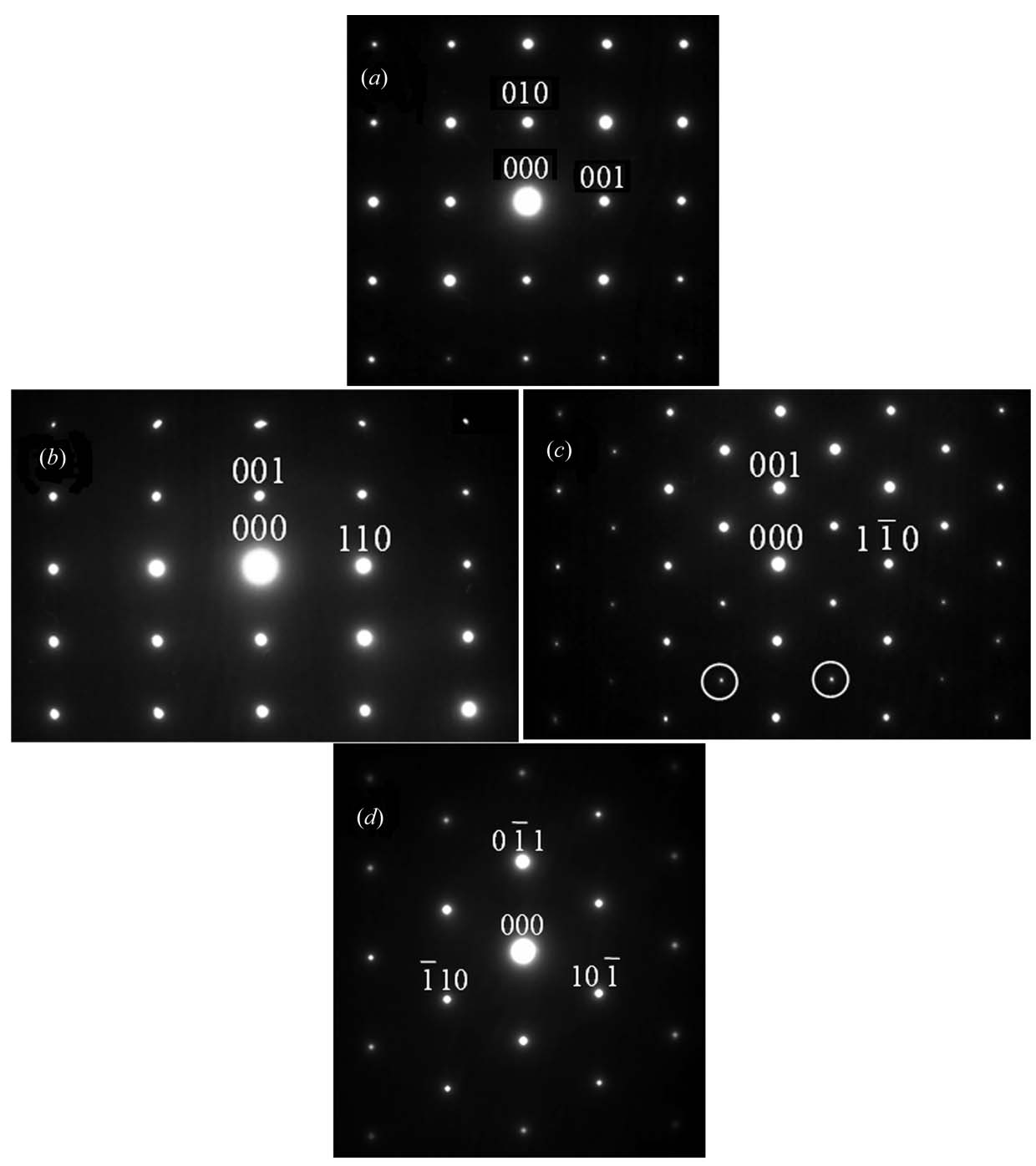

Figure 7

Electron diffraction patterns obtained from a sample of $\mathrm{BiFeO}_{3}$. These are arbitrarily indexed with zone axes $(a)$ [100], $(b)$ [110], $(c)$ [110] and $(d)$ [111]. Superlattice reflections arising from antiphase tilting are ringed.

\section{Mixed tilt systems}

Four distinct tilt systems exist that exhibit a combination of in-phase and antiphase tilting; $a^{0} b^{-} c^{+}$, $a^{-} a^{-} c^{+}, a^{-} b^{-} c^{+}$and $a^{+} a^{+} c^{-}$. All of these exhibit the reflections expected from the antiphase and in-phase tilt systems separately, but additional reflections (hereafter referred to as 'concert' reflections) caused by the combination of inphase and antiphase tilting are also observed at $\frac{1}{2}\{$ oee $\}$ positions in some $\langle 100\rangle$ and $\langle 110\rangle$ ZADPs for all four systems. Reflections are also generated with the general form $\frac{1}{2}\{$ ooe $\}$ in some of these systems, while the $a^{0} b^{-} c^{+}$system exhibits additional $\frac{1}{2}(o o o)$ reflections.

Concert reflections have been noted in neutron diffraction patterns of tilted perovskites (e.g. Howard et al., 2000). Reaney et al. (1994) associate reflections in the $\frac{1}{2}\{e e o\}$ positions with antiparallel shifts of the $A$-site species in a particular direction, but the intensity of these reflections also includes a contribution from the oxygen displacements. We include a kinematical treatment of these tilt systems, followed by a qualitative treatment assuming multiple scattering. 
$a^{+}$produces $\frac{1}{2}$ (oee) reflections with $\frac{1}{2}(h 00)$ reflections absent.

$b^{+}$produces $\frac{1}{2}(e o e)$ reflections with $\frac{1}{2}(0 k 0)$ reflections absent.

$c^{+}$produces $\frac{1}{2}(e e o)$ reflections with $\frac{1}{2}(00 l)$ reflections absent.

Two axes tilted in-phase produce $\frac{1}{2}($ oee $), \frac{1}{2}($ eoe $)$ and $\frac{1}{2}(e e o)$ reflections with $\frac{1}{2}(h 00), \frac{1}{2}(0 k 0)$ and $\frac{1}{2}(00 l)$

reflections absent, respectively.

In addition to these conditions, the reflections are also absent from the ZADP obtained parallel to the antiphase tilt axis. More precisely

$a^{0} b^{-} c^{+}: k \neq 0$.

$a^{-} b^{-} c^{+}$: no additional conditions.

$a^{-} a^{-} c+: h-k \neq 0$ (or $h^{+} k \neq 0$, depending on the sense

of antiphase tilt).

$a^{+} a^{+} c^{-}: h \neq \pm k$.

Reflections of the general type $\frac{1}{2}\{o o e\}$ are produced as follows

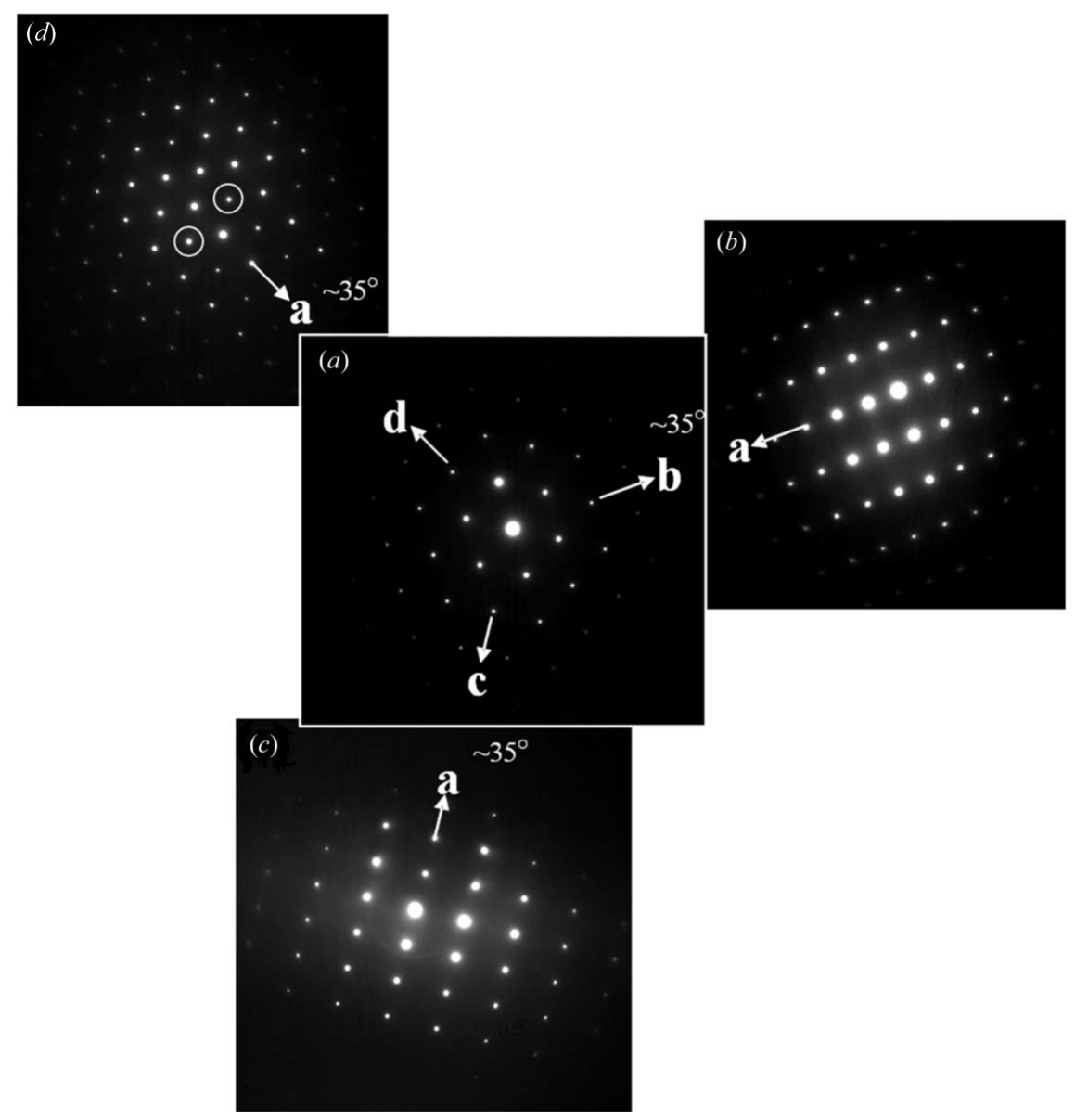

Figure 8

Results of tilt experiment for $\mathrm{BiFeO}_{3}$. (a) The central $\langle 111\rangle$ ZADP, (b) and (c) $\langle 110\rangle$ ZADPs without superstructure reflections, $(d)\langle 110\rangle$ ZADP with superstructure reflections (ringed). The labelled arrows indicate the orientational relationship between these diffraction patterns. $a^{+}$produces $\frac{1}{2}($ eoo $)$ reflections.

$b^{+}$produces $\frac{1}{2}($ oeo $)$ reflections.

$c^{+}$produces $\frac{1}{2}($ ooe $)$ reflections.

Two axes tilted in-phase produces $\frac{1}{2}($ ooe $), \frac{1}{2}($ oeo $)$ and $\frac{1}{2}($ eoo $)$ reflections.

These are indistinguishable from those generated solely by inphase tilting, although in some mixed-tilt systems there are differences in the conditions for absences. These are listed below

$$
\begin{aligned}
& a^{0} b^{-} c^{+}: \frac{1}{2}(h \bar{h} 0) \text { and } \frac{1}{2}(h h 0) \text { absent. } \\
& a^{-} b^{-} c^{+}: \text {No additional conditions. } \\
& a^{-} a^{-} c^{+}: h \neq \pm k \\
& a^{+} a^{+} c^{-}: \text {No additional conditions. }
\end{aligned}
$$

Finally, in the $a^{0} b^{-} c^{+}$system, $\frac{1}{2}\{o o o\}$ reflections are generated with no absences and as such will appear in every $\langle 110\rangle$ ZADP.

The application of multiple scattering to the kinematical simulation results in a distribution of the superstructure reflections around the various ZADPs. Table 8 lists the reflections that are allowed for the mixed tilt systems and the ZADPs in which they are allowed to appear.

The $\frac{1}{2}\{$ oee $\}$ reflections do not appear in solely in-phase or antiphase tilt systems and are therefore a useful indicator of mixed tilting. However, a note of caution should be sounded, as these reflections may also be created by antiparallel cation displacements leading to doubling along $\langle 100\rangle$ directions and so one should be careful if relying solely on these as proof of mixed tilting. However, the data in Table 8 simplify distinguishing between the systems $a^{-} b^{+} a^{-}$and $a^{0} b^{-} c^{+}$(space groups Pnma and Cmcm, respectively) - a problem for many researchers, particularly in the $(\mathrm{Ca}, \mathrm{Sr}) \mathrm{TiO}_{3}$ solid solution (e.g. Ball et al., 1998; Ranjan et al., 1999).

\section{Various notes of caution:}

In addition to tilting, there are two other sources of superstructure reflections; the antiparallel displacement of cations and the ordering of chemical species, such as dissimilar $A$ - or $B$-site cations and oxygen vacancies (Reaney, 1996). The superstructure reflections generated by antiparallel cation displacements (e.g. in antiferroelectric compounds) depend on the polarization axes and the repeat distances and as such are considered far too extensive to include here. Ordering of 
Table 8

List of superstructure reflections present in ZADPs for mixed tilt systems.

\begin{tabular}{lllll}
\hline Zone axis & $a^{0} b^{-} c^{+}$ & $a^{-} b^{-} c^{+}$ & $a^{-} a^{-} c^{+}$ & $a^{+} a^{+} c^{-}$ \\
\hline$[100]$ & $\frac{1}{2}($ eeo $)$ & $\frac{1}{2}($ eeo $)$ & $\frac{1}{2}($ eeo $)$ & $\frac{1}{2}($ eeo $)+\frac{1}{2}($ eoe $)+\frac{1}{2}($ eoo $)$ \\
{$[010]$} & - & $\frac{1}{2}($ eeo $)$ & $\frac{1}{2}($ eeo $)$ & $\frac{1}{2}($ eeo $)+\frac{1}{2}($ oee $)+\frac{1}{2}($ oeo $)$ \\
{$[001]$} & $\frac{1}{2}($ ooe $)$ & $\frac{1}{2}($ ooe $)$ & $\frac{1}{2}($ ooe $)$ & $\frac{1}{2}($ ooe $)$ \\
{$[110]$} & $\frac{1}{2}($ ooo $)+\frac{1}{2}($ ooe $)+\frac{1}{2}($ eeo $)$ & $\frac{1}{2}($ ooo $)+\frac{1}{2}($ ooe $)+\frac{1}{2}($ eeo $)$ & $\frac{1}{2}($ ooo $)$ & $\frac{1}{2}($ ooe $)$ \\
{$[110]$} & $\frac{1}{2}($ ooo $)+\frac{1}{2}($ ooe $)+\frac{1}{2}($ eeo $)$ & $\frac{1}{2}($ ooo $)+\frac{1}{2}($ ooe $)+\frac{1}{2}(e e o)$ & $\frac{1}{2}($ eeo $)$ & $\frac{1}{2}($ ooe $)$ \\
{$[101]$} & $\frac{1}{2}($ ooo $)$ & $\frac{1}{2}($ ooo $)$ & $\frac{1}{2}($ ooo $)$ & $\frac{1}{2}($ ooo $)+\frac{1}{2}($ oeo $)+\frac{1}{2}($ eoe $)$ \\
{$[101]$} & $\frac{1}{2}($ ooo $)$ & $\frac{1}{2}($ ooo $)$ & $\frac{1}{2}($ ooo $)$ & $\frac{1}{2}($ ooo $)+\frac{1}{2}($ oeo $)+\frac{1}{2}($ eoe $)$ \\
{$[011]$} & $\frac{1}{2}($ oooo $)$ & $\frac{1}{2}($ ooo $)+\frac{1}{2}($ eoo $)+\frac{1}{2}($ oee $)$ & $\frac{1}{2}($ ooo $)+\frac{1}{2}($ eoo $)+\frac{1}{2}($ oee $)$ \\
{$[011]$} & $\frac{1}{2}($ ooo $)$ & $\frac{1}{2}($ ooo $)$ & $\frac{1}{2}($ ooe $)$ & $\frac{1}{2}\{$ ooe $\}$ \\
$\langle 111\rangle$ & $\frac{1}{2}($ ooe $)$ & $\frac{1}{2}($ ooe $)$ & &
\end{tabular}

the repeat distance, subject to the condition $\frac{1}{y}(h+k+l)=n$ for ordering on the (111) planes. For example, $\mathrm{BaZn}_{1 / 3} \mathrm{Ta}_{2 / 3} \mathrm{O}_{3}$ exhibits 2:1 ordering, resulting in $y=3$ (Jacobson et al., 1976), although the reflections generated by ordering cannot be confused with reflections arising from tilting. However, problems may arise where 1:1 ordering exists. In this case, $\frac{1}{2}\{o o o\}$ reflections will appear in all $\langle 110\rangle$ ZADPs and mask any information concerning the presence or nature of antiphase tilting. In these cases, it is down to the individual concerned to decide

chemical species generally takes place on close-packed $\{111\}$ planes and will generate reflections at $\frac{1}{y}\{h k l\}$ positions in those ZADPs obtained parallel to the close-packed planes, where $y$ is an integer describing the number of close-packed planes in whether the species present are likely to order in this manner or whether the tolerance factor is of an appropriate value for antiphase tilting to occur (Reaney et al., 1994). In addition, as Lufaso \& Woodward (2001) point out, certain tilt systems such
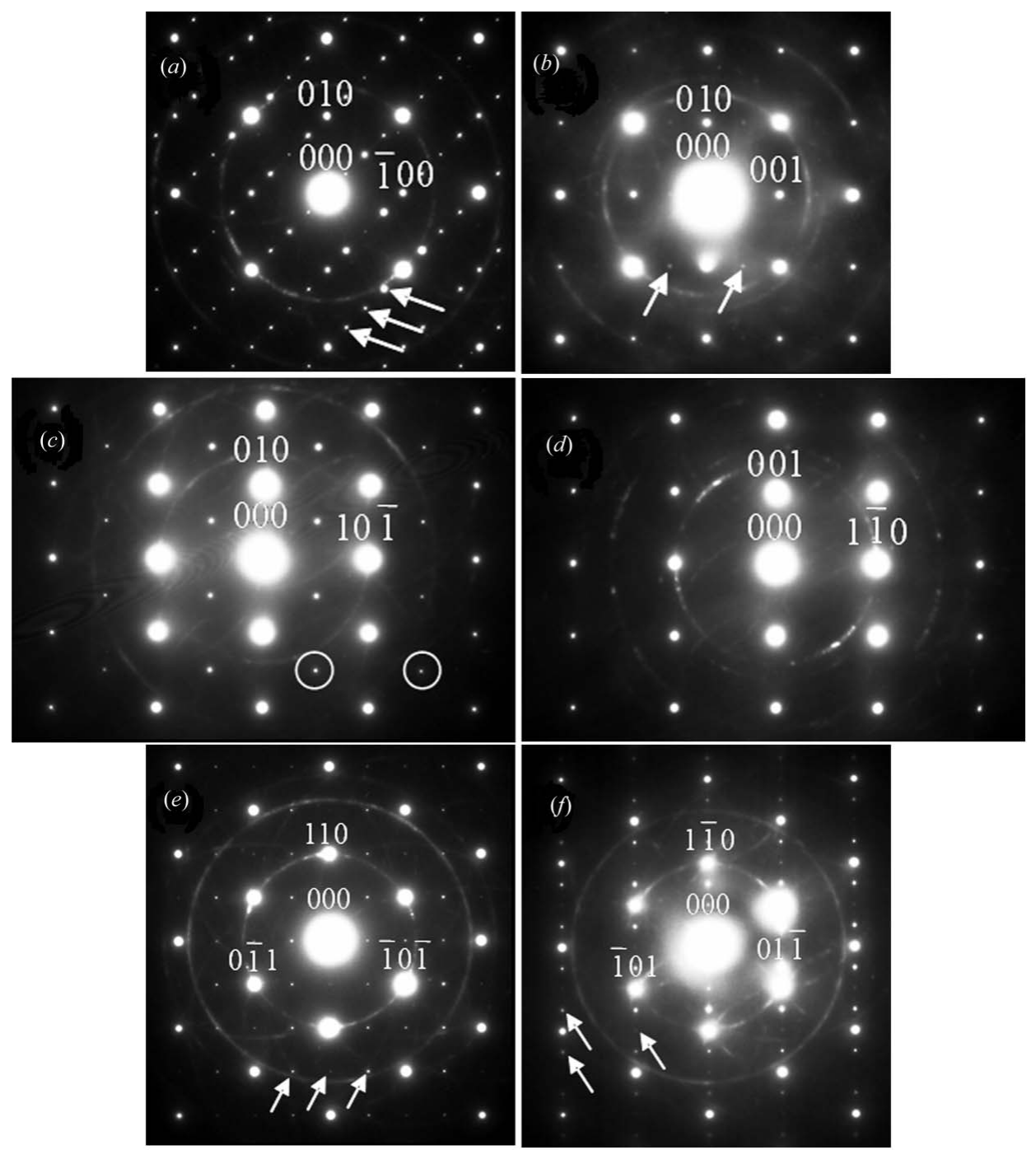

Figure 9

Diffraction patterns obtained from $\mathrm{PbZrO}_{3}$ sample with zone axes chosen as $(a)$ [001], (b) [100], $(c)$ [101], $(d)$ [110], $(e)$ [1111] and $(f)$ [111]. The superstructure spots relating to antiphase tilting are ringed. All other superstructure reflections, associated with other distortive mechanisms, are arrowed. as $a^{+} a^{+} a^{+}$have multiple $A$-site geometries and are stabilized by having multiple, ordered, $A$-site cations. Thus, ordering may be implicit in certain tilt systems and ordering reflections inextricably linked with the reflections associated with the tilt system, although in $\mathrm{Ca}_{3} \mathrm{CuTi}_{4} \mathrm{O}_{12}\left(a^{+} a^{+} a^{+}\right)$, the bodycentred ordering of $\mathrm{Ca}$ and $\mathrm{Cu}$ in $\mathrm{Ca}_{3} \mathrm{CuTi}_{4} \mathrm{O}_{12}$ produces exactly the same reflections as the tilt system (Propach, 1977).

Another important point is that the details published here show what reflections are allowed, but do not mean that the intensity of a superstructure reflection will be sufficient for it to be resolved from the background. Some concert reflections are expected to be particularly weak and great care should be taken to confirm their presence or absence. Similarly, the intensity of tilting reflections is directly related to the amplitude of tilt, and if this is extremely small, the spots may not be clearly visible.

All of the conditions listed depend on the electron diffraction patterns being obtained from single domains. In many cases, the electron diffraction patterns described above may be derived by the superposition of diffraction patterns from several adjacent domains. It is a necessity of using the data published here that diffraction is obtained from single domains. In some samples, the 
existence of fine-scale twinning may make this difficult or impossible. In such cases, it may be possible to employ convergent beam or micro-diffraction techniques to obtain diffraction patterns from the individual domains of the twinning region.

\section{Examples}

\section{1. $\mathrm{CaTiO}_{3}$}

Diffraction patterns obtained from a TEM sample of $\mathrm{CaTiO}_{3}$ are shown in Fig. 6 (for details of fabrication, see Bagshaw et al., 2003). These represent all the possible variants of the $\langle 100\rangle,\langle 110\rangle$ and $\langle 111\rangle$ ZADPs found in $\mathrm{CaTiO}_{3}$. The superstructure reflections in Figs. $6(b)$ and $(e)$ are indicative of in-phase tilting about a single axis, chosen arbitrarily as [010]. This limits the tilt system to one of four possibilities: $a^{0} b^{+} a^{0}$, $a^{0} b^{+} c^{-}, a^{-} b^{+} c^{-}$or $a^{-} b^{+} a^{-}$. The superstructure reflections in Fig. 6(c) indicate antiphase tilting, eliminating $a^{0} b^{+} a^{0}$. The diffraction pattern in Fig. $6(d)$ is not observed with either $a^{0} b^{+} c^{-}$or $a^{-} b^{+} c^{-}$tilt systems. This leaves $a^{-} b^{+} a^{-}$as the only possible tilt system. This corresponds to the space group Pnma

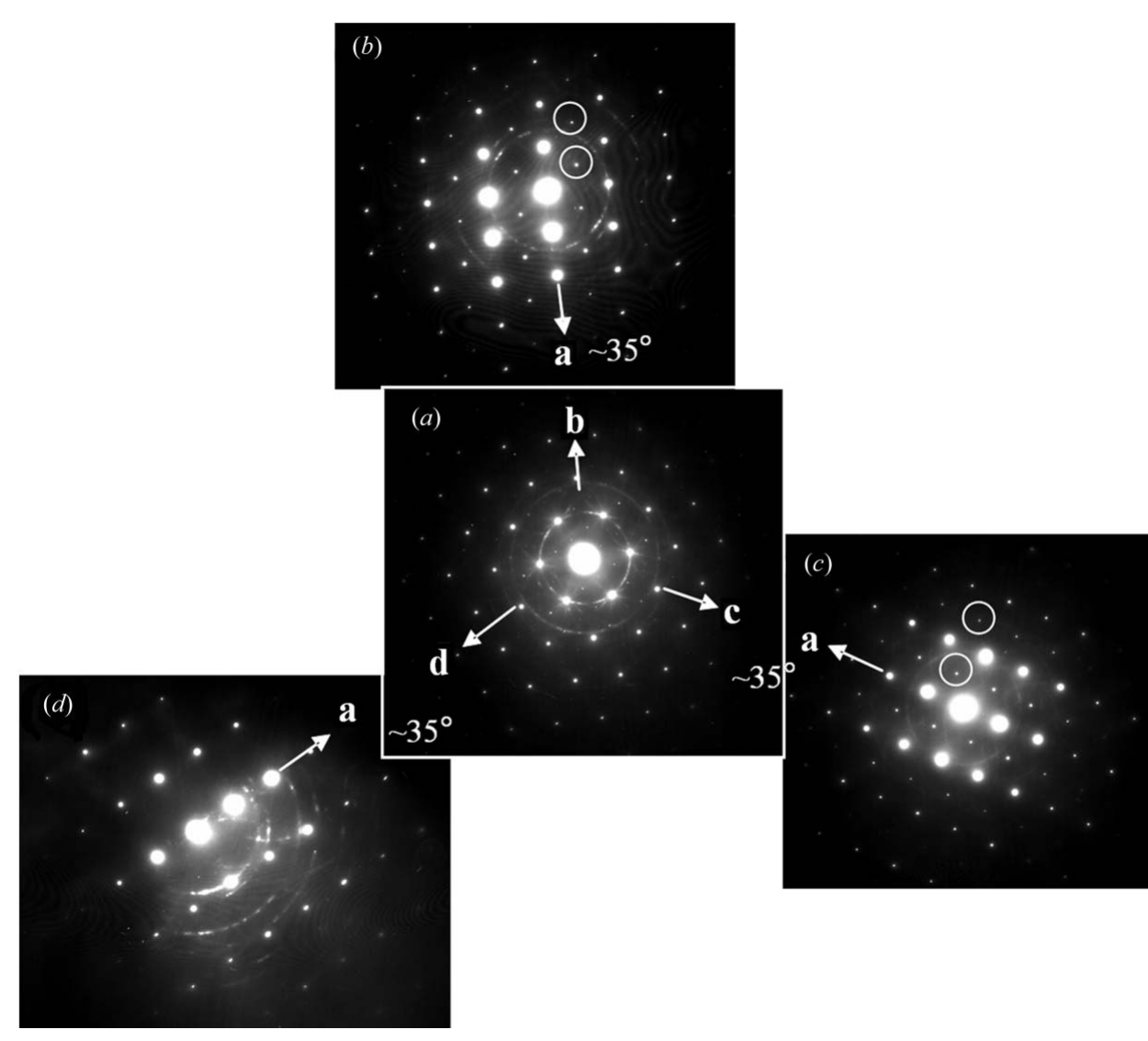

Figure 10

Results of the first tilt experiment for $\mathrm{PbZrO}_{3}$. (a) The central $\langle 111\rangle$ ZADP, (b) and (c) $\langle 110\rangle$ ZADPs with superstructure reflections (ringed), $(d)\langle 110\rangle$ ZADP without superstructure reflections. The labelled arrows indicate the orientational relationship between these diffraction patterns.
(Glazer, 1972), which is the recognized space group for this compound.

\section{2. $\mathrm{BiFeO}_{3}$}

Diffraction patterns obtained from a TEM sample of $\mathrm{BiFeO}_{3}$ are shown in Fig. 7 and represent all variants of the $\langle 100\rangle,\langle 110\rangle$ and $\langle 111\rangle$ ZADPs that may be obtained from this material (for fabrication details, see Woodward et al., 2003). The absence of superstructure reflections from the $\langle 100\rangle$ and 〈111〉 ZADPs (Figs. $7 a$ and $c$ ) indicates that in-phase tilting is not present. The presence of $\frac{1}{2}\{o o o\}$ superstructure reflections in some $\langle 110\rangle$ ZADPs demonstrates that antiphase tilting is present, but the absence of these reflections from some $\langle 110\rangle$ ZADPs eliminates $a^{0} b^{-} c^{-}$and $a^{-} b^{-} c^{-}$tilt systems (see Table 2 ). The data in Table 3 are now used to relate the occurrence of superstructure reflections around a $\langle 111\rangle$ zone with the tilt system. Fig. 8 shows the result of one such experiment conducted on a single domain in the $\mathrm{BiFeO}_{3}$ sample. Two of the three $\langle 110\rangle$ ZADPs do not exhibit superstructure reflections, but one does. Table 3 shows that this arrangement corresponds only to the $a^{-} a^{-} a^{-}$tilt system. The space group associated with this system is $R \overline{3} c$ (Glazer, 1972). The recognized space group for this material is $R 3 c$, a subgroup of $R \overline{3} c$ obtained through the loss of the centre of symmetry which occurs due to ferroelectric cation displacements.

\section{3. $\mathrm{PbZrO}_{3}$}

$\mathrm{PbZrO}_{3}$ was made by a standard mixed-oxide processing route. Powders were milled and then reacted for $4 \mathrm{~h}$ at $1023 \mathrm{~K}$ followed by sintering for $3 \mathrm{~h}$ at $1525 \mathrm{~K}$. Further details of the fabrication may be found elsewhere (Knudsen, 2002).

Identification of the tilt system present in $\mathrm{PbZrO}_{3}$ is complicated by the presence of many additional superstructure reflections which arise as a result of a particular arrangement of antiparallel ionic displacements (see Figs. $9 a, b, e$ and $f$ ). Some of these reflections are clearly visible, but some that appear in simulations of the diffraction patterns are so weak in practice that they do not appear in the diffraction patterns included here. In addition, some of these appear in positions that could be associated with types of tilting. However, with careful treatment, it is still possible to use the methods presented here to exclude the majority of tilt systems. 
The superimposed polycrystalline ring patterns arise from surface damage of the $\mathrm{PbZrO}_{3}$ during milling. This has been previously observed and is not considered to affect the crystallographic data from the perovskite phase (Reaney et al., 1997).

Some $\frac{1}{2}\{$ ooe $\}$ reflections are observed in some $\langle 100\rangle$ and $\langle 111\rangle$ ZADPs (Figs. $9 a$ and $f$ ) that might be associated with inphase tilting. However, the rules of in-phase tilting demand that all $\langle 111\rangle$ ZADPs contain $\frac{1}{2}\{$ ooe $\}$ reflections. As this is not the case (Fig. 9e), in-phase tilting is not present. The $\langle 110\rangle$ ZADPs exist both with and without $\frac{1}{2}\{0 o o\}$ superstructure reflections, demonstrating, as with $\mathrm{BiFeO}_{3}$, that antiphase tilting is present, but that tilt systems $a^{0} b^{-} c^{-}$and $a^{-} b^{-} c^{-}$are excluded. The tilt experiment presented for $\mathrm{BiFeO}_{3}$ in the previous section is performed around the first $\langle 111\rangle$ variant of $\mathrm{PbZrO}_{3}$ (Fig. 10) and reveals that two out of three $\langle 110\rangle$ ZADPs (Figs. $10 b$ and $c$ ) contain $\frac{1}{2}\{o o o\}$ superstructure reflections, thus eliminating the $a^{-} a^{-} a^{-}$tilt system and leaving $a^{0} a^{0} c^{-}, a^{0} b^{-} b^{-}$and $a^{-} b^{-} b^{-}$as the only possibilities. A second tilt experiment (Fig. 11) performed around the second $\langle 111\rangle$

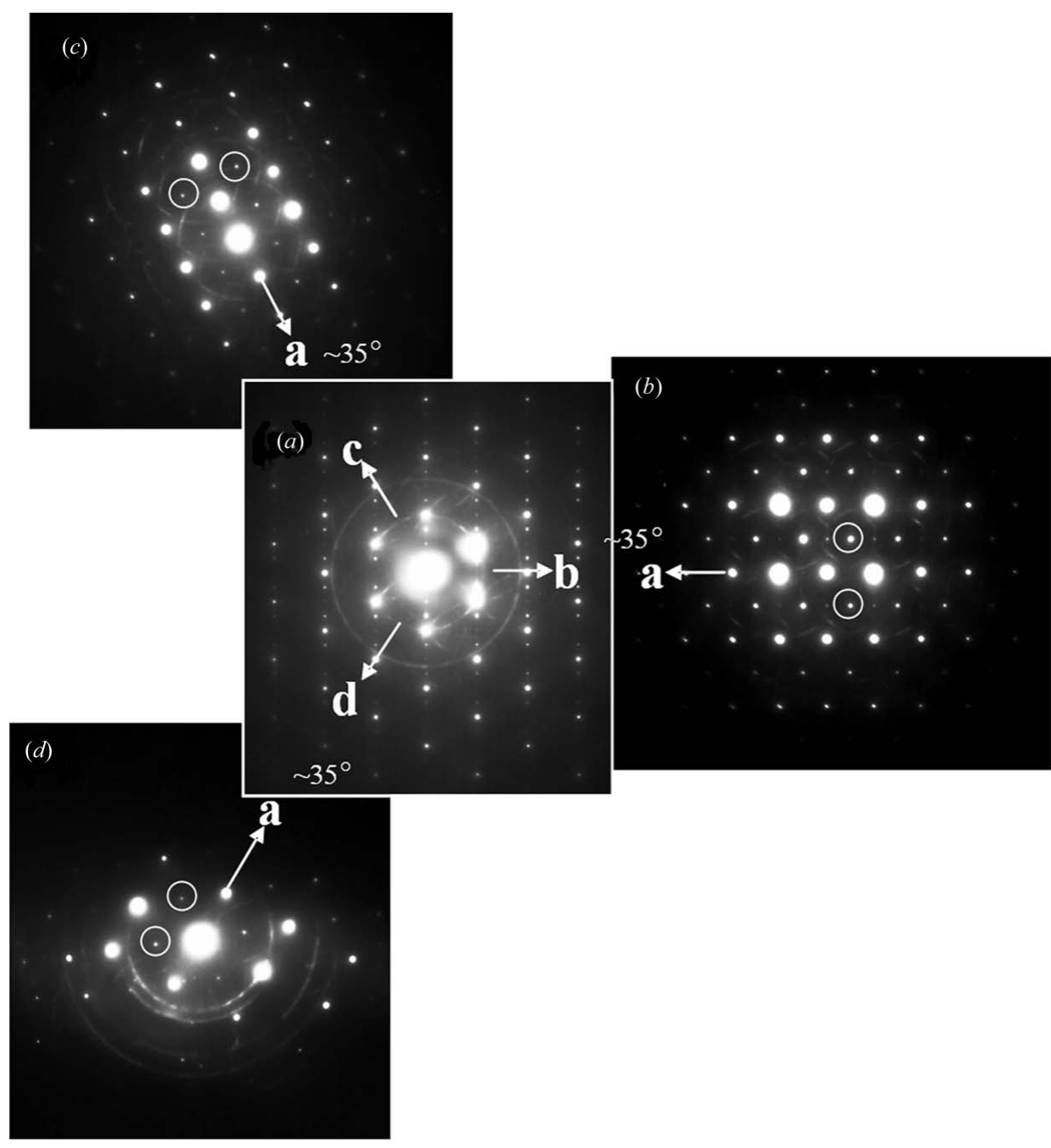

Figure 11

Results of the second tilt experiment for $\mathrm{PbZrO}_{3}$. (a) The central $\langle 111\rangle \mathrm{ZADP},(b),(c)$ and $(d)\langle 110\rangle$ ZADPs with superstructure reflections (ringed). variant reveals that all three $\langle 110\rangle$ ZADPs contain $\frac{1}{2}\{o o o\}$ reflections, eliminating the $a^{0} a^{0} c^{-}$tilt system.

Distinguishing between $a^{0} b^{-} b^{-}$and $a^{-} b^{-} b^{-}$tilt systems relies on observing differences in intensity of the superstructure reflections found in different zones. As these intensities are affected by more than just the tilt angle or axis, electron diffraction is not the most reliable way to determine the tilt system and in this case the tilt system is left with two possible choices. However, the tilt system is known from structural refinements against $\mathrm{X}$-ray diffraction patterns to be $a^{0} b^{-} b^{-}$(Glazer et al., 1993).

\section{4. $\mathrm{Na}_{1 / 2} \mathrm{Bi}_{1 / 2} \mathrm{TiO}_{3}$ (NBT; in situ hot-stage study)}

NBT ceramics were made by a standard oxide/carbonate processing route. Stoichiometrically mixed powders were calcined for $2 \mathrm{~h}$ at $1023 \mathrm{~K}$ and sintered for $2 \mathrm{~h}$ at $1448 \mathrm{~K}$. Neutron diffraction experiments conducted by Jones \& Thomas $(2000,2002)$ have shown that NBT has the tilt system $a^{0} a^{0} c^{+}$between 673 and $773 \mathrm{~K}$.

Diffraction patterns were obtained from a thinned ceramic sample of NBT heated to $713 \mathrm{~K}$. The variants of the $\langle 100\rangle,\langle 110\rangle$ and 〈111〉 ZADPs obtained from this sample are shown in Fig. 12. The $\frac{1}{2}($ ooe $)$ reflections seen in Fig. 12(d) show the presence of in-phase tilting about a single axis, confirmed by the superstructure reflections seen in some, but not all $\langle 100\rangle$ ZADPs (Figs. $12 a$ and $b$ ) and consistent with the $a^{0} a^{0} c^{+}$tilt system. Unexpectedly though, all recorded $\langle 110\rangle$ ZADPs exhibit extremely weak $\frac{1}{2}(o o o)$ reflections which do not immediately appear to be consistent with the proposed tilt system. However, the extreme weakness of these reflections, relative to the in-phase tilt reflections, gives reasonable grounds to question whether these really relate to macroscopic antiphase tilting. It is known that for NBT, two different structures - one with in-phase tilting and one with antiphase tilting - may coexist over certain tmperature ranges, and although this is not expected at the temperature used, only a small volume fraction of this structure would be required to produce the $\frac{1}{2}(o o o)$ spots seen. Alternatively, these reflections may relate to very weak, localized ordering of the $A$-site cations. This may be more likely as the different 
valences of $\mathrm{Na}^{+}$and $\mathrm{Bi}^{3+}$ provide an appropriate driving force for ordering, but no other reports of ordering are known for NBT. Ultimately, however, these data indicate that the macroscopic tilt system is $a^{0} a^{0} c^{+}$, although small deviations from this tilt system cannot be completely ruled out.

\section{Conclusions}

All distinct tilt systems for perovskites listed by Howard \& Stokes $(1998,2002)$ have been simulated and the effects on the resulting allowed reflections and electron diffraction patterns listed with the intention of simplifying the allocation of tilt systems and ultimately space groups to perovskites. Particular attention has been paid to the use of selected-area electron diffraction as a key laboratory-based tool for solving tilt systems. The inclusion of tables indicating the structural
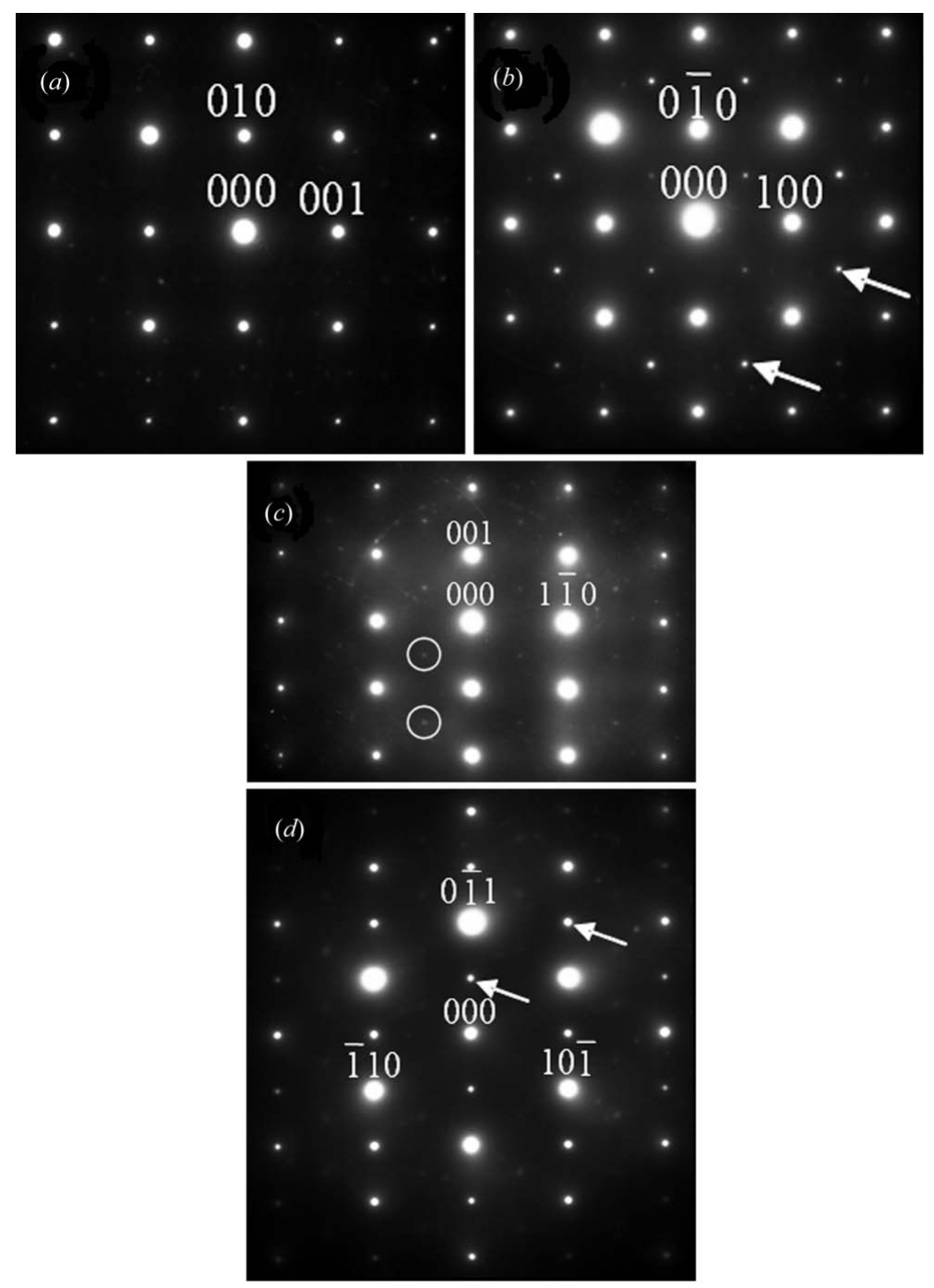

Figure 12

Diffraction patterns obtained from an NBT sample at $713 \mathrm{~K}$. These are indexed with zone axes $(a)$ [100], $(b)$ [001], (c) [110] and $(d)$ [111]. Superlattice reflections arising from in-phase tilting are arrowed. Weak superlattice reflections possibly arising from antiphase tilting are ringed. significance of the appearance and arrangements of superstructure reflections will enable future researchers to make the fullest use of selected-area electron diffraction as a technique for investigating tilting of perovskites. The use of this technique has been adequately demonstrated for several tilt systems for known compounds and structures.

Thanks to Pat Woodward (Ohio State University, USA) and Mike Lufaso (University of South Carolina, USA) for the use of their computer programs and their enthusiasm. For ceramic processing, the authors wish to thank Rachel Wallace and Heath Bagshaw. We appreciate the help of Peter Korgul and Peter Kenway at the Sorby Centre for Electron Microscopy, University of Sheffield, and the Manchester Materials Science Centre, University of Manchester, respectively, for their patience and tutoring of electron microscopy. This work was partly funded as part of the Engineering and Physical Science Research Council (EPSRC) portfolio award, GR/S60037/01 within the Ceramics and Composites Laboratory, Engineering Materials, University of Sheffield.

\section{References}

Bagshaw, H., Iddles, D., Quimby, R. \& Reaney, I. M. (2003). J. Eur. Ceram. Soc. 23, 2435-2441.

Ball, C. J., Begg, B. D., Cookson, D. J., Thorogood, G. J. \& Vance, E. R. (1998). J. Solid State Chem. 139, 238-247.

Glazer, A. M. (1972). Acta Cryst. B28, 3384-3392.

Glazer, A. M. (1975). Acta Cryst. A31, 756-762.

Glazer, A. M., Roleder, K. \& Dec, J. (1993). Acta Cryst. B49, 846-852.

Goldschmidt, V. M. (1926). Geochemische verteilungsgesetze der elemente, I Mater. Naturvid. K1. no 2. Oslo: Skrifter Norske Videskaps-Akad.

Howard, C. J., Knight, K. S., Kennedy, B. J. \& Kisi, E. H. (2000). J. Phys. Condens. Matter, 12, L677-L683.

Howard, C. J. \& Stokes, H. T. (1998). Acta Cryst. B54, 782-789.

Howard, C. J. \& Stokes, H. T. (2002). Acta Cryst. B58, 565.

Jacobson, A. J., Collins, B. M. \& Fender, B. E. F. (1976). Acta Cryst. B32, 1083-1087.

Jones, G. O. \& Thomas, P. A. (2000). Acta Cryst. B56, 426-430.

Jones, G. O. \& Thomas, P. A. (2002). Acta Cryst. B58, 168-178.

Knudsen, J. (2002). Thesis. University of Sheffield.

Knudsen, J., Woodward, D. I. \& Reaney, I. M. (2003). J. Mater. Res. 18, 262-271.

Leinenweber, K. \& Parise, J. (1995). J. Solid State Chem. 114, 277-281.

Lufaso, M. W. \& Woodward, P. M. (2001). Acta Cryst. B57, 725-738.

O'Keeffe, M. \& Hyde, B. G. (1977). Acta Cryst. B33, 3802-3813.

Noheda, B., Gonzalo, J. A., Cross, L. E., Guo, R., Park, S.-E., Cox, D. E. \& Shirane, G. (2000). Phys. Rev. B, 61, 8687-8695. 
Propach, V. (1977). Z. Anorg. Allg. Chem. 435, 161-171.

Ranjan, R., Pandey, D., Siruguri, V., Krishna, P. S. R. \& Paranjpe, S. K. (1999). J. Phys. Condens. Matter, 11, 2233-2246.

Reaney, I. M. (1996). Proc. Electroceramics V, Portugal, pp. 441446.

Reaney, I. M., Colla, E. L. \& Setter, N. (1994). Jpn. J. Appl. Phys. 33, 3984-3990.

Reaney, I. M., Glazounov, A., Chu, F., Bell, A. \& Setter, N. (1997). Brit. Ceram. Trans. 96, 217-224.
Woodward, P. M. (1997a). Acta Cryst. B53, 32-43.

Woodward, P. M. (1997b). J. Appl. Cryst. 30, 206-207.

Woodward, P. M. (1997c). Acta Cryst. B53, 44-66.

Woodward, D. I., Reaney, I. M., Eitel, R. E. \& Randall, C. A. (2003). J. Appl. Phys. 94, 3313-3318.

Wul, B. M. \& Goldman, I. M. (1945). Comput. R. Acad. Sci. USSR, 46, 139-142.

Zhao, Y., Weidner, D. J., Parise, J. B. \& Cox, D. E. (1993). Phys. Earth Planet. Inter. 76, 17-34. 